\title{
Clinical Outcome of Arthroscopic Treatment of Anterior Ankle Pathologies
}

\author{
Kutsi Tuncer ${ }^{1}$, Mehmet Köse ${ }^{1}$, Murat Topal ${ }^{2}$, Eyüp Şenocak ${ }^{3}$, Ömer Selim Yıldırım ${ }^{1}$ \\ ${ }^{1}$ Ataturk University, Faculty of Medicine, Department of Orthopaedics and Traumatology, Erzurum, Turkey \\ ${ }^{2}$ Kastamonu University, Faculty of Medicine, Department of Orthopaedics and Traumatology, Kastamonu, Turkey \\ ${ }^{3}$ Erzurum Regional Training and Research Hospital, Clinic of Orthopaedics and Traumatology, Erzurum, Turkey
}

Received: 03 March 2019, Accepted 26 April 2019, Published online: 28 August 2019

(C) Ordu University Institute of Health Sciences, Turkey, 2019

\begin{abstract}
Objective: The ankles that are the main organs cause standing over the earth can be damaged by different reasons. The ankles become painful with the problems like impingement syndrome that is soft tissue hipertrofies, osteochondral defects that is cartilage damages, traumatic or degenerative arthropaties, intraarticular tumoral tissues and some problems like that. These painful ankles can be treated with conservative methods. If it is impossible to treat painful ankles with conservative way it can be treated by minimal invasive ankle arthroscopy that is cheaper and much more satisfied, of treatment.

Methods: We aim to evaluate the results and effectiveness of arthroscopic treatment of the patient who has the forefoot disorders. Also, we hope to compare the advantages, difficulties and probable complications of the arthroscopic treatment according to conservative therapy and to investigate the factors that can effect the clinical results. The 40 patients who were practiced and operated with ankle arthroscopy who was followed at least one year were compared statistically with pre-op and post-op condition. By this way the effectiveness of arthroscopy for the patients who have anterior ankle problems was determined and the main factors that effect the results were investigated. According to the result of statistical analyses preop and postop AOFAS and NPS score avarages were found clear different to each other.

Results: The ankle arthroscopy that is practised by experienced surgeons with an appropriate procedure after a good clinical practise and a proper indication causes a high patient satisfaction and it causes less and temporary complications. Because of all these reasons we believe that the ankle arthroscopy can be practised by the appropriate surgeon to the appropriate patient population.

Conclusion: We believe that the outcomes of ankle arthroscopy performed properly by experienced hands after determining the patient population who were accurately diagnosed by careful clinical examination and necessary imaging methods are greatly satisfactory, and the acceptability is high thanks to complications being less and usually transient.

Key words: Ankle arthroscopy, ankle pathologies, anterolateral impingement, osteochondral defect, ankle arthrosis
\end{abstract}

Suggested Citation: Tuncer K, Köse M, Topal M, Senocak E, Yıldırım OS. Clinical Outcome of Arthroscopic Treatment of Anterior Ankle Pathologies. Middle Black Sea Journal of Health Science, 2019; 5(2): 54-62.

Address for correspondence/reprints:

Kutsi Tuncer

Telephone number: +90 (505) 5571337

E-mail: drkutsi@hotmail.com

DOI: $\quad 10.19127 / \mathrm{mbsjohs.534920}$
Note: This study was presented as a verbal presentation at the 28th National Orthopedics Congress in Antalya TURKEY, at 30.10.2018-04.11.2018 


\section{Introduction}

In Turkey, it is known that the first arthroscopy was performed in 1977 by Veli Lök, the first publication of a cadaver study on ankle arthroscopy was published by Pinar et al. (1989), and the first clinical trial was conducted by Aydin (1991)

Ankle arthroscopy is an endoscopic method which allows the visualization of the joint without the necessity to perform arthrotomy or malleolar osteotomy, and performing surgical intervention in the joint and the surrounding soft tissues (Aydin and Gokkus, 2013). It is a minimally invasive technique which is applied by placing the necessary devices through two incisions of approximately. 0.5 $\mathrm{cm}$. The reasons why ankle arthroscopy is preferred include the visualization of all intraarticular structures and the possibility of intervention, low post-operative morbidity, and easy and rapid rehabilitation (Aydin, 1996).

Patients with anterior ankle pathologies whom were admitted to our clinic between 2011-2013 were divided into three groups (anterolateral impingement, osteochondral lesions, arthrosis) and had been evaluated regarding the need for arthroscopic treatment. Beneficence of arthroscopic management for the patients have been evaluated with comparison of the preoperative and postoperative AOFAS and NPS scores. 40 patients who had could have been properly followed up, out of 52 patients have been included to the study. We do think that this study will make significant contribution to the literature regarding the beneficence of arthroscopic surgery for the anterior ankle pathologies.

\section{Methods}

Patients who admitted to our clinic with ankle problem between July 2011 and July 2013 were assessed using AOFAS scores after a detailed history taking and physical examination in the outpatient clinic. Patients with the possible need for arthroscopic treatment were referred to thesis resident. All patients were examined by same physician and the problems were determined. Arthro-MRI was taken and interpreted by an experienced radiologist in many patients who were deemed necessary to undergo MR to examine the problems in the region more clearly. Patients with reflex sympathic dystrophy syndrome (sudeck atrophy), excessive edema around the joint, peripheral vascular pathology, open wound in operation site, tinea pedis, or cellulitis underwent a therapy program based on their underlying condition and were referred to the related clinics. Patients with anterior ankle pain for at least 3 months who did not benefit or even worsened using conservative methods and physical therapy were informed about arthroscopic treatment and recommended to undergo arthroscopy if there is a possibility to find a solution using arthroscopic treatment.

Three groups were formed from patients with anterior ankle pathologies including impingement syndrome (including the ones of osseous and soft tissue origin), osteochondral talus defect, and ankle arthrosis due to various reasons, and included into the study. The radiological appearance of the lesions of the patients with osteochondral defect were grouped before the operation based on the classification specified by Berndt and Harty.

All patients were assessed before the operation using AOFAS (American Orthopedic Foot \& Ankle Society) ankle-hindfoot scale and NPS (Numerical Pain Scale). Following the last vital sign control in the ward, the patients received antibiotic (IV, $1 \mathrm{gr}$ cefazolin). Important anatomic structures were drawn using skin pen in the operating room. Using fourth toe traction and foot inversion, effort was made to observe superficial peroneal nerve. The patients received anesthesia of their choice after being informed. The patients were laid down on their back in a position as described by Parisen. A pillow was placed under the hip of the operation side (toes pointing towards the ceiling). Additionally, another pillow was placed on the cruris midline of the operation side to facilitate ankle manipulation and use the instruments effectively. The foot was in a position extending out of the table. The operation table was adjusted to a level so that the surgeon can manipulate the foot freely with his belly. Tourniquet was not used in any of the patients.

Four mm 30 degree scope which is used for knee arthroscopy was used. Manual traction device made of sterilized gauze was wrapped around the ankle and foot and prepared for the procedure. 10$15 \mathrm{cc}$ of isotonic injection was injected into the joint through anteromedial soft point. The degree of easiness of the fluid flow was observed, and the ankle was observed to go into dorsiflexion with the ongoing fluid injection. The syringe was removed by leaving the needle inside, and fluid was observed to flow out.

Fluid entry to the joint was applied under control by Y-pump using $3000 \mathrm{~mL}$ of isotonic with $0,5 \mathrm{mg}$ of adrenaline inside. During the case, shaver 
and RF device were kept ready to use. As the operation period did not reach 2 hours in any of the patients, no additional antibiotic was required during the operation. Postoperative splint-plaster cast application was not necessary in any of the patients.

They were told they can walk for necessary activities for the first 3 days and gradually increase their walking without overdoing for the first 3 weeks. Osteochondral defect pathology patients with stage II and stage III lesion underwent debridement together with arthroscopic microfracture operation, and patients with stage IV lesion underwent curettage and arthroscopic microfracture operation after fragment removal. All patients who underwent microfracture operation were requested to start ankle exercise on the postoperative second day and recommended to avoid putting load on that ankle for three weeks. After three weeks, exercises for increasing joint range of motion and muscle strength were recommended for three months by putting partial load on the ankle. Sports activities were forbidden for 6 months.

Unless another problem arises, the patients were requested to return for 3-week, 3-month, 6-month and 1-year follow-up visits. In the follow-up visits, all patients were assessed using AOFAS anklehindfoot score and NPS score. Patients with a postoperative follow-up period less than a year were excluded from the study. After the completion of the study, the patients were grouped, and their AOFAS and NPS score progression was evaluated. As patients who underwent arthroscopic debridement and grafting due to mass in talus, and septic arthritis irrigation-debridement during the study were not suitable for any of the 3 groups or other diseases, and as their number was not as high as to make an evaluation, they were excluded from the evaluation.

\section{Results}

In descriptive statistics, numerical data were expressed as mean and standard deviation, and categorical data as number and percentage. For the comparison of disease groups and pathologies, Chi-Square and Fisher's Exact tests were used. For the comparison of the group scores, Kruskal-Wallis test was used. For the comparison of preoperative, and postoperative 3-month and 1-year data of the patients (for repeated samples), ANOVA test and Wilcoxon test were used. A p value of $<0.05$ was considered to be significant.

The study included 40 patients. The mean age of the cases was $44.1 \pm 12.3$ years. Twenty-six of the patients $(65 \%)$ were males, and $14(35 \%)$ were females. The pathological side which underwent operation was right ankle in $18(45 \%)$ patients and left ankle in 22 $(55 \%)$. The weights of the patients were grouped as thin, normal, overweight and obese. No significant relationship was found in the comparison of sides, sex and weights with disease groups.

The patients were classified by 3 disease groups and 8 different pathologies. The following table shows the percentage of the patients by group and pathology (Table 1).

Table 1. Patient distribution by pathology

\begin{tabular}{lcc}
\hline & Number & Percentage \\
\hline Anterior Impingement & 5 & 12,5 \\
Anterior Osseous & 3 & 7,5 \\
Impingement & 7 & 17,5 \\
Anterolateral Impingement & 1 & 2,5 \\
Anteromedial Impingement & 8 & 20,0 \\
Medial OCD & 3 & 7,5 \\
Anterolateral OCD & 10 & 25,0 \\
Traumatic Arthritis & 3 & 7,5 \\
Degenerative Arthritis & 40 & 100,0 \\
\hline Total &
\end{tabular}

The patient's preoperative, postoperative 3month, postoperative 1-years and last follow-up visit AOFAS values, and preoperative NPS and postoperative last follow-up visit NPS values were checked. Significant changes were observed in postoperative AOFAS values (Table 2). 
Table 2. The mean AOFAS and NPS scores of the patients in pre- and postoperative period

\begin{tabular}{lccccc}
\hline & N & Mean & SD & Min & Max \\
\hline $\begin{array}{l}\text { Preoperative } \\
\text { AOFAS Score }\end{array}$ & 40 & 52,95 & 13,521 & 24 & 72 \\
$\begin{array}{l}\text { Postoperative 3- } \\
\text { Month AOFAS }\end{array}$ & 40 & 75,30 & 9,090 & 47 & 87 \\
$\begin{array}{l}\text { Score } \\
\text { Postoperative 1- }\end{array}$ & 40 & 74,50 & 11,239 & 41 & 89 \\
$\begin{array}{l}\text { Year AOFAS } \\
\text { Score }\end{array}$ & & & & & \\
$\begin{array}{l}\text { Last Follow-Up } \\
\text { Visit AOFAS }\end{array}$ & 40 & 73,37 & 11,758 & 41 & 89 \\
$\begin{array}{l}\text { Score } \\
\text { Preoperative NPS }\end{array}$ & 40 & 5,25 & 1,171 & 3 & 8 \\
$\begin{array}{l}\text { Score } \\
\text { Last Follow-Up } \\
\text { Visit NPS Score }\end{array}$ & 40 & 2,35 & 1,099 & 1 & 5 \\
\hline \multicolumn{1}{l}{ Min: Minimum; Max: Maximum; SD: Std Deviation }
\end{tabular}

One-way ANOVA was planned to test the distribution of AOFAS and NPS scores in patient groups. Histogram graphs were studied to evaluate the data distribution. Some data was observed to be non-normally distributed. See below for histogram graphs (Graphs 1-2-3-4-5-6).

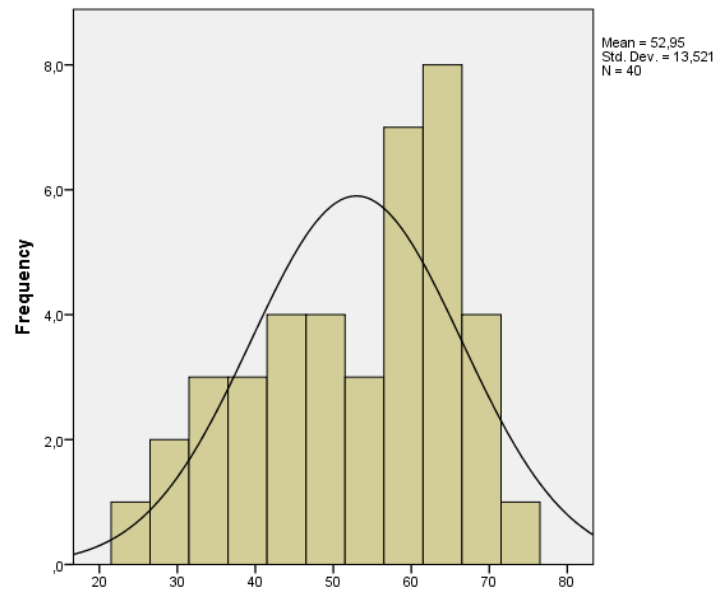

Graphs 1. Preop AOFAS score

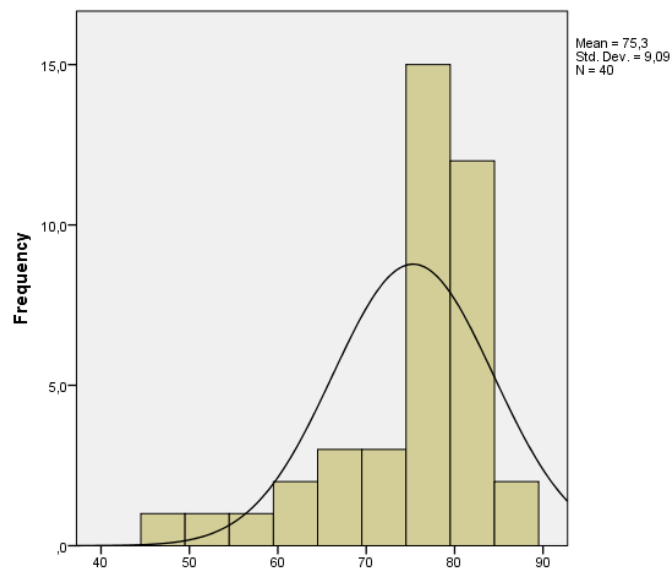

Graphs 2. Postop 3th month AOFAS score

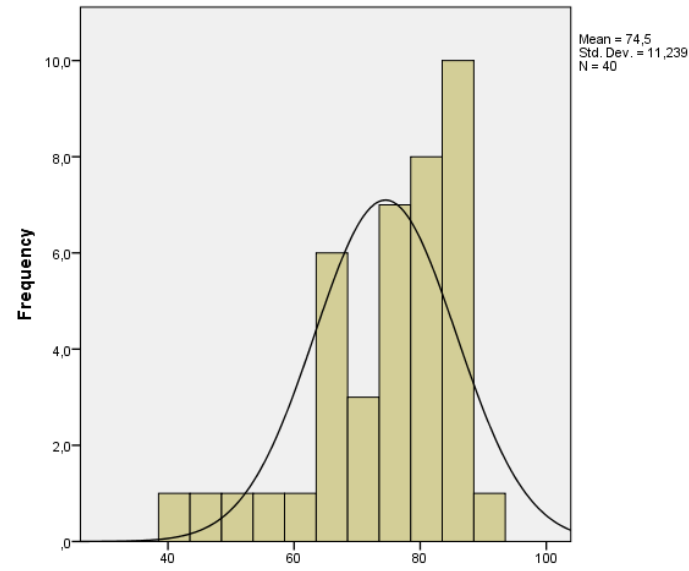

Graphs 3. Postop 1st year AOFAS score

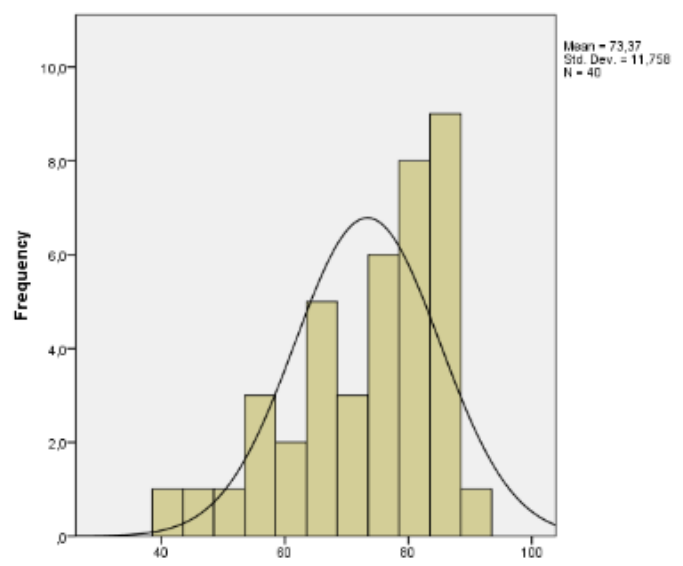

Graphs 4. Last control AOFAS score

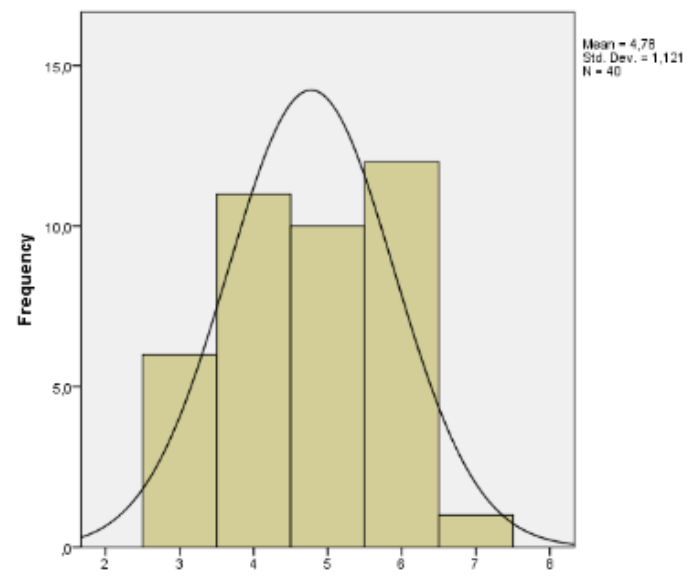

Graphs 5. NPS score before operation 


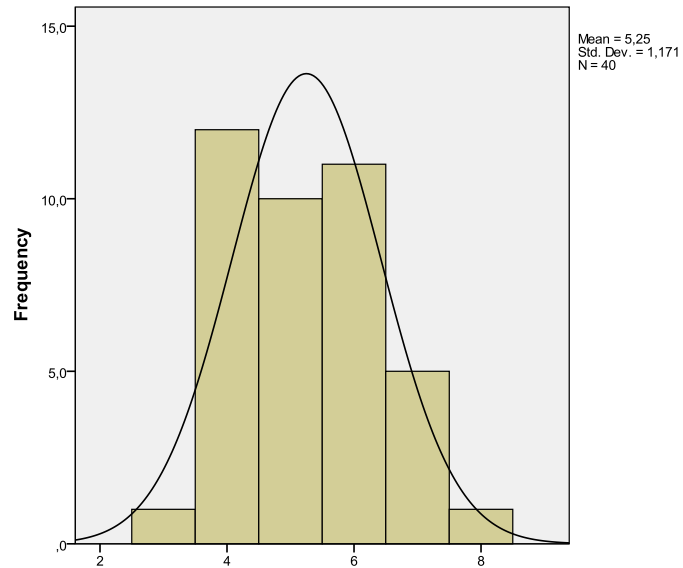

Graphs 6. NPS score on the last check

Therewith, being a non-parametric alternative of one-way ANOVA, Kruskal-Wallis test was used. Preoperative AOFAS, postoperative 3-month AOFAS, postoperative 1-year AOFAS, and last follow-up visit AOFAS scores of the patients were observed to be significantly different. For the NPS scores, no significant difference was found between preoperative and last follow-up visit values of the patient groups.

The patients' AOFAS scores were evaluated in preoperative period, and at postoperative month 3 and year 1 . These values were evaluated using ANOVA test for repeated samples. As $p$ was $<0.05$ in the Mauchly's ANOVA, sphericity hypothesis could not be established, and Greenhouse-Geisser value in Tests of Within- Subjects Effects table was shown. Significant difference between the repeated measures of the scores. $\mathrm{P}<0.001$ (Graph 7)

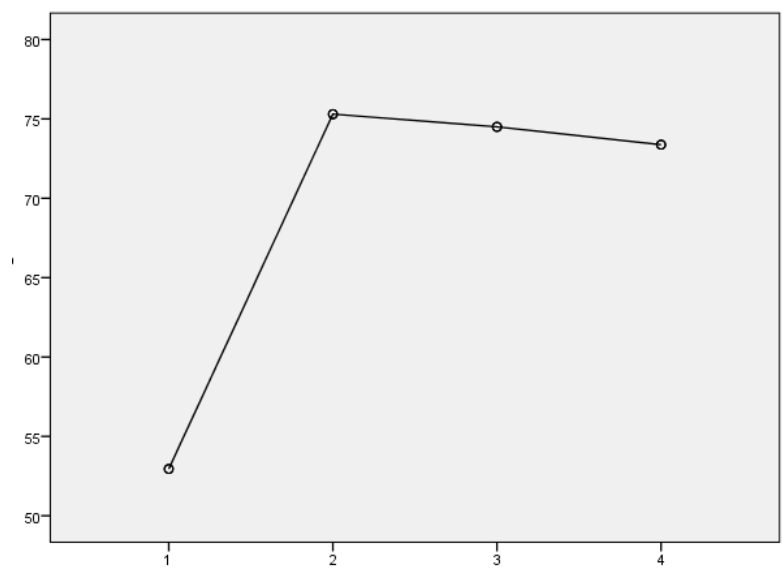

Graph 7. Estimated Marginal Means of AOFAS (1: Preop, 2: Postop 3th month, 3: Postop 1st year, 4: Last control AOFAS)
As the preoperative and last follow-up visit NPS scores of the patients were non-normally distributed, Wilcoxon test which is the nonparametric alternative of the t-test for dependent samples. Significant difference was observed between the pre- and postoperative scores. $\mathrm{P}<0.001$

The total follow-up period of the patients was 17.60 months (min. 12, max. 30 months). The mean preoperative AOFAS score was $52.95( \pm 13.521$, min. 24, max. 72). The mean postoperative 3-month AOFAS score was $75.30( \pm 9.09$, min. 47 , max. 87); the mean postoperative 1-year AOFAS score was 74.50 ( \pm 11.239 , min. 41 , max. 89$)$; the mean last follow-up visit AOFAS score was $73.38( \pm 11.758$, min. 41, max. 89). The mean preoperative NPS score was $5.25( \pm 1.171$, min. 3, max. 8) and the mean last follow-up visit NPS score was 2.35 $( \pm 1.099$, min. 1, max. 5).

When the patients were evaluated by disease subgroups, out of patients with impingement syndrome, 7 were diagnosed with anterolateral impingement in preoperative period, 1 with anteromedial impingement, and 5 with anterior impingement due to synovial hypertrophy in both regions. The diagnoses were confirmed postoperatively. Three patients had osseous anterior impingement. Thereby, the mean age of 16 impingement patients included into the study was calculated to be 45.63 (min. 32, max. 72) years. The total follow-up period of the patients was 18 months (min. 12, max. 30 months). The mean preoperative AOFAS score was $58.94( \pm 9.248$, min. 36, max. 69). The mean postoperative 3-month AOFAS score was $79.31( \pm 5.82$, min. 66 , max. 87$)$; the mean postoperative 1-year AOFAS score was $78.25( \pm 8.56$, min. 52, max. 89$)$; the mean last follow-up visit AOFAS score was $77.75( \pm 8.67$, min. 58, max. 89). The mean preoperative NPS score was $4.88( \pm 1.20$, min. 4 , max. 8$)$ and the mean last follow-up visit NPS score was 2.06 $( \pm 0.998$, min. 1, max. 5).

In the second group consisting of 11 patients with osteochondral defect, 8 had medial and 3 had anterolateral pathology. The mean age of the patients was 43.55 (min.23, max.70). Male/female ratio was 8/3. The total follow-up period of the patients was 17.55 months (min. 12, max. 27 months). The mean preoperative AOFAS score was $57( \pm 11.045$, min. 40, max. 69). The mean postoperative 3-month AOFAS score was 79.55 $( \pm 2,382$, min. 76 , max. 84$)$; the mean postoperative 1 -year AOFAS score was $81( \pm 5.34$, min. 68 , max. 86); the mean last follow-up visit AOFAS score 
was $79.82( \pm 5.67$, min. 66 , max. 84$)$. The mean preoperative NPS score was $5.09( \pm 0.83$, min. 4, max. 6) and the mean last follow-up visit NPS score was 1.91 ( \pm 0.944 , $\min .1$, max. 4$)$.

All 5 patients at stage II according to the radiological classification of Berndt and Harty had good clinical outcomes according to the clinical outcome classification of Berndt and Harty. Two of 4 patients at stage III had good, and the other 2 had moderate outcomes. Two patients with stage IV osteochondral lesion had moderate outcome. In subjective assessments, all patients were found to be satisfied with the ankle function in their daily activities. When evaluating the clinical outcomes, postoperative MRI was requested only in 4 patients. A complete correlation was not observed between clinical and MRI findings of these patients. The patients returned their daily life activities 6 weeks to 3 months after the surgery. Completely returning to sports activities was allowed 6 months after the surgery.

In the last group consisting of 13 patients with arthrosis, only 3 had arthrosis due to degenerative reasons and 10 due to traumatic reasons. The mean age of the patients was 42.85 (min. 25, max. 68 months) years. The total follow-up period of the patients was 17.15 months (min. 12, max. 30 months). These patients had a mean preoperative AOFAS score of $42.15( \pm 14.017$, min. 24, max. 72), and the mean postoperative 3-month AOFAS score was $66.77( \pm 10.22$, min. 47 , $\max .78)$; the mean postoperative 1-year AOFAS score was 64.38 $( \pm 11.25$, min. 41 , max. 82$)$; the mean last follow-up visit AOFAS score was $62.54( \pm 11.73$, min. 41, max. 82). The mean preoperative NPS score was $5.85( \pm 1.21$, min. 3, max. 7) and the mean last follow-up visit NPS score was 3.08 ( \pm 1.38 , min. 2 , max. 5). In this group, one patient with arthrosis had a low AOFAS score at postoperative month 3 due to severe pain at 3-month follow-up visit because of a loose body falling in the joint due to trauma approximately 3 months after the operation. After the removal of the loose body, same patient was evaluated to have an AOFAS score of 82 in the next follow-up visit.

Three patients developed postoperative complications. One of them had transient superficial peroneal nerve paralysis, 1 had complaint worsening, and the other one described locking ankle while walking. The male OCD patient with superficial peroneal nerve paralysis was followed up using Vitamin B supplement. His complaints regressed starting from the first month and at month 3 , he had no complaint at all. As the female impingement syndrome patient with complaint worsening stated that her pain is more than before when standing up in the first follow-up visits, additional imaging was requested. However, no pathology was detected that may cause pain. Conservative treatment methods were recommended. In the 3-month follow-up visit, the patient stated that her pain level is at the same level before the operation. The condition was unchanged in 1-year follow-up visit. The remaining patient with complication who had no complaint in the early follow-up visits was a female patient with impingement syndrome. Stating that she has to stop for a few seconds due to locking ankles while walking, the complaints started approximately 6 months after the operation. There was no pathology found in the additional imaging which can explain the symptoms. As the study only included patients with 1-year follow-up, the patient who developed reflex sympathic dystrophy in postoperative period and did not return for follow-up visits after month 3 was excluded from the study.

The complication rate seen in 40 patients was $7.5 \%$ (3 patients); and the complication rates by group were $12.5 \%$ ( 2 patients) in 16 patients with impingement syndrome, $9.09 \%$ (1 patient) in 11 patients with osteochondral defect, and $0 \% \quad(0$ patients) in 13 patients with arthrosis. It should be remembered that transient superficial peroneal nerve paralysis improved without any sequela in approximately 3 months, and 2 patients with worsening pain and locking ankles while walking were considered to have complication without any detectable medical pathology. The only major complication seen in 52 patients including 12 patients who were excluded from the study due to various reasons (lost-to-follow-up, follow-up period less than 1 year) was reflex sympathic dystrophy seen in 1 patient.

Among the patients included into the study; one patient who underwent operation for degenerative arthritis had FMF and possibly missed old Tillaux fracture. While this patient has significantly improved AOFAS value and life quality in the early postoperative period, the complaints in the region were worsened again after another trauma. Another patient who underwent operation for traumatic arthritis had bilateral ankle arthrosis due to old trauma and stated that he cannot carry out the postoperative resting recommendations as we requested.

Increase of AOFAS scores from the mean of 
52.95 to 74.50 at the first year follow up and decrease of NPS scores from the mean of 5,25 to 2,35 at the last follow up visit can be interpreted as significant beneficence. Also minimally invasive technique of the procedure, low rate of complications, shorter period of hospitalization and returning to work are major advantages of the technique. Groups had also been evaluated separately. AOFAS score has increased from 57 to 81 and NPS score has decreased from 5 to 1,91 in the group with osteochondral lesions. AOFAS score has increased from 59 to 78,2 and NPS score has decreased from 4,88 to 2,06 in the group with anterior impingement. AOFAS score has increased from 42 to 64 and NPS score has decreased from 5,85 to 3,08 in the group with arthrosis. Evaluating the difference between the first year follow up and preoperative AOFAS scores the group had the most beneficence was the arthrosis group (\%54).

\section{Discussion}

Being gradually more used in the last 25 years for ankle pathologies, ankle arthroscopy became an important alternative for open surgeries as it is a minimally invasive procedure. The reasons why ankle arthroscopy is preferred include the visualization of all intraarticular structures and the possibility of intervention, low post-operative morbidity, easy and rapid rehabilitation, and the patients being able to return their work, social and sports activities as soon as possible (Aydin,1996; Tosun and Yilmaz, 2009). It is a sensitive matter that the portals to be opened for arthroscopy procedure allow the most effective way to perform the procedure and also avoid damaging important anatomic structures. Thereby, it becomes more favorable method compared to open surgical interventions, and the complications of open surgical procedure can be reduced.

Arthroscopy procedure is usually performed under tourniquet and in aqueous setting (Aydin and Gokkus, 2013). The basis of seeing tourniquet use as a rule is undoubtedly image quality. Conditions such as acute hemorrhagic synovitis and pigmented villonodular synovitis also support this opinion (Lawrence and Albert, 2011).

There are also surgeons who do not wish their patient go through tourniquet pain and try to avoid DVT risk caused by tourniquet by performing arthroscopy applications without tourniquet (Tecimer at al., 1995). Hence, a recent publication stating that tourniquet use does not provide any extra benefit was published by Zaidi et al. (Zaidi at el., 2014). However, there are also publications that cannot state tourniquet use is unnecessary but exhibit impartial attitude due to its risks (Smith and Hing, 2010). We did not use tourniquet in our cases, and we believe that image quality for procedure was obtained by adding adrenalin inside the fluid to provide vasoconstriction. Because we did not encounter a problem caused by not using tourniquet in any of our ankle arthroscopy cases. The necessary intraarticular fluid pressure can be adjusted by hanging the irrigation solution up, and using pump Y-catheter or arthropump pressureadjusted electronic system (Aydın and Gokkus, 2013).

For ankle arthroscopy, standard imaging system used for the knee and knee arthroscopy instruments would be enough. Optically, standard $4 \mathrm{~mm} \mathrm{30-}$ degree optic is adequate. However, for convenience, short-barrel (14 $\mathrm{mm})$ optic and sleeves can be used. Microfracture apparatus at different angles designed for ankle, ring curettes, guides for drilling using special Kirschner wire (Ferkel's retrograde drilling guide), Hembfling's intraarticular distractor, fine shaver tips, Boehler and radiofrequency (RF) probes are also used (Aydin, 1996; Aydin, 2009; Aydın and Gökkuş, 2013). We perform our ankle arthroscopy procedures without using any special instrument and by completely using knee arthroscopy instruments in our clinic.

In a study in 2009, Glazebrook et al. investigated the outcomes of different treatments for ankle pathologies using Pubmed database, and graded the generally accepted treatment methods by acceptability. In the study named 'Evidence-based indications for ankle arthroscopy', fair evidencebased literature (Grade B) is present to support using ankle arthroscopy for the treatment of 'impingement syndrome' and osteochondral lesions, and ankle arthrodesis. Ankle arthroscopy for ankle instability, septic arthritis, arthrofibrosis and removing free bodies is only supported by lowquality evidence (Grade C). Ankle arthroscopy treatment for isolated bone impingement is not effective, and thereby, this indication is not recommended (Grade C opposing). Lastly, they concluded that evidence-based literature to support or reject the hypothesis that arthroscopy is beneficial in the management of synovitis and fracture is not adequate (Class I) (Glazebrook, 2009). While ankle arthroscopy is definitely contraindicated in periarticular soft tissue infection (e.g., cellulitis, chronic or acute open wound, 
periarticular dermatitis) and degenerative arthritis in several joints, it is relatively contraindicated in moderate arthritis (narrowing which restricts joint movement), edema, peripheral vascular diseases, peripheral neuropathy, reflex sympathic dystrophy/complex regional pain syndrome, and toe infections.

Complications predominantly consist of nerve injuries (approx. 50-60\%). And majority of nerve injuries (approx. 50-60\%) is at superficial peroneal nerve (Ferkel et al., 1996). Among the complication rate stated to be $9 \%$ by Ferkel in their series consisting of 612 cases, the rate of neurological complications was high, $49 \%$. Out of these 27 cases, $56 \%$ had injury to superficial peroneal nerve, $22 \%$ to sural nerve, and $18 \%$ to long saphenous nerve. Deep peroneal nerve injury has only been reported in 1 case $(4 \%)$. These injuries have been stated to be observed especially in cases where anterocentral or posteromedial portals, and invasive distraction devices are used (Ferkel et al., 1996). There are many studies stating that complications rates dramatically drop when non-invasive distractors are used (Sprague et al., 1989; Ferkel et al., 1996; Deng et al., 2011; Zengerink and van Dijk, 2012).

As most of the complications are related with portals, the anatomy of the region should be known very well and routine portals should be used (Aydin, 1996). It is recommended to visualize and mark superficial peroneal nerve which is at high risk for injury during anterolateral portal opening by traction of the 4th toe, plantar flexion and inversion (in $1 / 3$ of the patients) before starting the operation if possible; and in addition to this or if the nerve cannot be visualized by this method, it is known that the subcutaneous structures can be protected by entering through anteromedial portal and giving out light from inside (Ferkel et al., 1996; Lawrence and Albert, 2011).

As a nuance, when we put the foot in plantar flexion and inversion to mark the superficial peroneal nerve and then leave it back to neutral position, the nerve shifts to lateral side by $3.6 \mathrm{~mm}$ on average. And this leads to injury to the nerve which we marked to avoid injury during portal opening. It has been recommended to stay on the medial side of the mark to avoid this risk (Zengerink and van Dijk, 2012). In the light of this information, we tried to open anterolateral portal rigorously in every case. We believe the fact that superficial peroneal nerve injury, which has been reported at various rates in several publications and is almost the most common complication, was only seen in 1 of our cases is a result of our sensitivity.

Anatomic studies have demonstrated that anterior tibial artery passes close to anterolateral portal in $6.7 \%$ of the cases, and the complications such as pseudoaneurysm or vascular injury are caused by this closeness (Son et al., 2011).

There are inconclusive matters in postoperative period, and some authors state that they are important for complication control. While Ferkel recommended 1 week immobilization and prophylactic antibiotic use in postoperative period (Ferkel et al., 2001), van Dijk did not recommend postoperative antibiotic use and starts in case of superficial infection. He recommends the patients should start postoperative movement on the same day, put the foot in active dorsiflexion every few hours, put load on the foot as much as they can tolerate, and use the crutches for 4-5 days maximum (Zengerink and van Dijk, 2012). We recommended every patient we discharged to change the dressing every 3 days, suture removal on the 15th day, and to use prophylactic antibiotic during this period. Including the patients who refused or forgot using antibiotics, none of our cases had superficial or deep infection. We also believe that by trying to mobilize our patients as soon as possible, the regional perfusion was improved and the resistance to infections was increased. Excluding the patients who underwent microfracture operation due to chondral defect, we tried to apply a policy of putting load on the day after the operation and allowing active walking only 3 weeks after as much as possible.

\section{Conclusion}

We believe that the outcomes of ankle arthroscopy performed properly by experienced hands after determining the patient population who were accurately diagnosed by careful clinical examination and necessary imaging methods are greatly satisfactory, and the acceptability is high thanks to complications being less and usually transient. 
Ethics Committee Approval: Ethics committee approval was received for this study from Faculty of Medicine Clinical Research Ethics Committee of Ataturk University. B.30.2.ATA.0.01.00/93 30.05.2014

Peer-review: Externally peer-reviewed.

Author Contributions: Concept- KT, ÖSY; Design- KT, MT, EŞ; Supervision- KT, MK, ÖSY; Literature Review- KT, MT; Writing- KT, EŞ; Critical Review- KT, MK.

Conflict of Interest: No conflict of interest was declared by the authors.

Financial Disclosure: The authors declared that this study hasn't received no financial support.

\section{References}

Aydin A.T, Gokkus K: Ankle arthroscopy: indication and technique, Journal of TOTBID; 2013; 12: p. 134-141.

Aydin AT. Ankle arthroscopy (Indications, diagnostic and surgical arthroscopy), Acta Orthop Traumatol Turc 1996; 30: p. 470-483.

Aydin AT, Ankle arthroscopy. In: Aydin AT, Editor. Ankle arthroscopy. Antalya: Orkun Ozan Media Services INC. 2009; p. 42-44.

Aydin AT. Diagnostic and operative arthroscopy of the ankle. 1 st. Turkish Sports Traumatology, Arthroscopy and Knee Surgery Congress, İstanbul-Turkey:1991, 25-28 Sepl.

Ferkel RD, Heath DD, Guhl JF. Neurological complications of ankle arthroscopy. Arthroscopy. 1996; 12: p. 200-208.

Ferkel RD, Small HN, Gittins JE. Complications in foot and ankle arthroscopy. Clin Orthop Relat Res 2001; 391: p. 89-104.

Glazebrook MA, Ganapathy V, Bridge MA, Stone JW, Allard JP. Evidence-based indications for ankle arthroscopy. Arthroscopy. 2009; 25: p. 1478-90.

Lawrence AD, Albert AG. Current concepts in ankle arthroscopy. Podiatry today. 2011; 24(4): p:54-61.

Pinar H, Aydinok HC, Altunan AK: Arthroscopy of the cadaver ankle. Acta Orthop traum Turcic 1989; 23: p. 317-321.

Smith TO, Hing CB. The efficacy of the tourniquet in foot and ankle surgery? A systematic review and meta-analysis. Foot Ankle Surg. 2010; 16: p. 3-8.
Son KH, Cho JH, Lee JW, Kwack KS, Han SH. Is the anterior tibial artery safe during ankle arthroscopy?: Anatomic analysis of the anterior tibial artery at the ankle joint by magnetic resonance imaging. Am J Sports Med 2011; 39: p. $2452-56$.

Tecimer T, Yedek I, Bilgic E, Zaim E, Kılıckap C: Use tourniquet in extremity surgery, Acta Orthop Traumatol Turc 1995; 29: p. 172-176.

Tosun H.B, Y1lmaz E. The Results of microfracture method in the treatment of osteochondral lesions of the talus. Firat Medical Journal 2009; 14(3): p. $175-180$

Zaidi R, Hasan K, Sharma A, Cullen N, Singh D, Goldberg A. Ankle arthroscopy: a study of tourniquet versus no tourniquet: Foot Ankle Int. 2014; 35: p. 478-82.

Zengerink M, van Dijk CN. Complications in ankle arthroscopy. Knee Surg Sports Traumatol Arthrosc. 2012; 20: p. 1420-31. 